\title{
Communication Improvement by Analyzing Human Pupil Variation in Online Study
}

\author{
Ritai $\mathrm{Yu}^{1}$ \\ School of Computer Science and Technology \\ Hangzhou Dianzi University \\ Hangzhou, China \\ Guozhong Zhou ${ }^{2}$ \\ School of Computer Science and Technology \\ Hangzhou Dianzi University \\ Hangzhou, China
}

\author{
Baixi Xing* \\ School of Computer Science and Technology \\ Hangzhou Dianzi University \\ Hangzhou, China \\ sisyxing@gmail.com
}

\begin{abstract}
MOOC is increasingly popular widespread among education, however it makes changes on teaching mechanism, resulting in communication barrier, especially for discipline which is highly interactive and interdisciplinary. Here we propose the pitfall of MOOCs and would like to discuss the possible way of bridging the communication gap by a more "HCI" way, that using pupil variation recognition of student's studying status as a feedback for MOOCs teacher. Better communication could increase the experience of engaging and affecting for online course environment. In the experiment, SVM algorithm was validated to recognize the arousal-valence emotion states of the pupil diameter variation features, which provided an effective model for further mobile application development. By this means, we hope to improve the MOOCs education quality and also obtain useful data to make interesting exploration on MOOCs user behavior study.
\end{abstract}

Keywords-emotion recognition, support vector machine, pupil diameter, MOOCs

\section{INTRODUCTION}

MOOC has been a popular lecture form in education among universities. Most MOOCs used video lectures to transform knowledge, which overcomes restrictions of time and location of studying. However, it was surfaced by various challenges currently. The dropout rate of MOOCs was up to $90 \%$ due to reasons of understanding problem, lack of incentive and sense of helpless etc [8]. For MOOCs teaching, four main challenges were also concluded: 1. students' response deficiency [1] [3] [10]; 2. an experience of speaking into a vacuum with no feedback [1] [6] [14]; 3. time cost burden in course preparation [2] [9]; 4. difficulty of students' study quality evaluation [7]. Among them, you might find that three reasons were related to communication. Considering the characteristics of user behavior in MOOCs teaching and learning, that users are usually watching the interface in a very close distance (Figure 1), we would like to use pupil variation information captured by webcam or cellphone camera as a feedback for teachers.

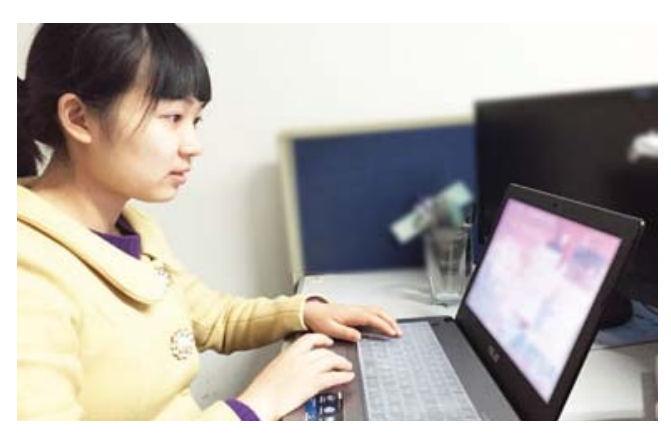

Fig. 1. MOOC Studying Behavior [4]

Eyes won't lie. True emotions and status could be revealed by eyes. The pupil will change according to different arousal of awareness and sensibility, since pupil diameter and reaction are innervated by both sympathetic and parasympathetic nerves. Therefore, the instant emotion and status of students could be recognized by analyzing pupil variation features. As to emotion and status recognition, we could recognize emotions from two aspects: the non-biological features and the bio-features. The former included music, text, image, video etc. The latter was the reaction of the body to the subjective emotion recognition, including heart rate, facial expressions, biological signal etc.

Comparing to the other bio-features, the facial expressions could have interference, while EMG, EEG, and ECG features are hard to collect for the huge amount of MOOCs user crowd which need specific wearable devices to detect. Pupil features are relatively easy to capture by camera. There were numbers of studies demonstrate that it is feasible to use pupil features to recognize people's status of concentration and emotion [5] [13] [11] [12]. The research procedure is shown in Figure 2. 


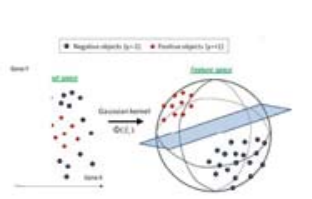

Student Status Recognition Modeling T

Machine Learning Algorithms

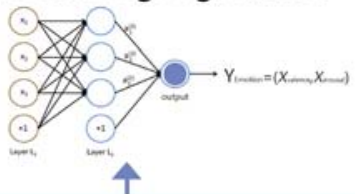

Pupil Variation Features Extraction
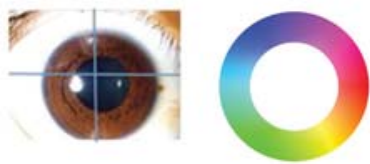

1.Pupil Dilation

2. Pupil Variation Rate 3. HSV RGB

Fig. 2. Pupil variation recognition research roadmap

\section{RELATED WORKS}

To our knowledge that many studies have reported that the pupil changes (including pupil diameter, expansion and contraction rate, color and light reflection characteristics) could reflect peoples emotional status and cognitive features.

Eye movement features were analyzed to acquire pupil variation information in different emotion status, explore the relationship between pupil features and emotion [5]. Timo Partala used 10 types of sound, including positive, negative and neutral, to make stimulates for studying the pupil size changes [13]. The experimental results showed that the pupil variation stimulated by positive and negative sound was significantly greater than the neutral voice stimuli, and they also found that female's pupil changes is more obvious, which proved that pupil variation could be used in assessment of instantaneous emotion changes. It was demonstrated by the study of Onorati $\mathrm{F}$. that pupil dilation rate varied by different emotions [12]. Matiko J.W. analyzed comprehensive data to identify emotion, including heart rate, skin conductance, pupil dilation, tone of voice, facial expression and electroencephalogram (EEG), that accuracy up to $63.52 \%$ was achieved [11].

Professor Eckhart of the University of Chicago American once organized a research experiment about pupil change and the result is that for different emotional state, the degree of change of the pupil is not the same. For example, when women see a picture on a mother holding a child, their pupil diameter will expand to $25 \%$ averagely. Compared to the micro expressions and heart rate, the change of pupil is the micro body movements, which is difficult to be controlled by our will. In that case, we can avoid the interference of expression and identify the person's real emotional state.

In addition, the changes of pupil is also used in lie-detect and medical research. By using the method of measuring pupillary changes, Dionisio [15] detected lying or not and then found $92 \%$ of 24 subjects' pupil in lie changed larger significantly than the when they were in truth. Trying to creating a model of a face (eyes, nose, mouth, etc.) to identify subtle fear, disgust and surprise, Avinash R.Vaidya's [16] experimental results show that through the eyes to recognize the most subtle emotions is the best. Therefore, according to the pupil changes, people can recognize people's real emotional state.

The existing studies showed that PD was varied due to emotion, pressure and stimulation, it could be an effective variable contributed to the recognition of students' status.

\section{EXPERIMENT}

We carried out the series of experiments in order to study the relationship between pupil features and emotions. Eventually we collected 30 individual pupil emotion data, and established a pupil characteristics database with emotional dimension.

\section{A: The Emotion Space}

As to biological evolution, emotions can be divided into two categories: basic emotion and compound emotion [17]. Wei-Chun Chiang used a hierarchical support vector machines (SVMs) to recognized and divided music emotions from the dynamics, the rhythm, the pitch and timbre aspects into four types that were happy, tensional, sad and peaceful[18]. Based on the intensity, polarization and similarity to divide emotion, R. Plutchik [19] divided emotions into eight aspects: ecstasy, vigilance, grief, surprise, rage, fear, acceptance and hatred, and then established a three-dimensional distribution. Ekman have proposed a classification of six basic emotions: anger, happiness, fear, surprise, disgust, sadness [20]. Janghoon Yang researched existing emotion space model to try to integrate existing emotion models and found that there is a fundamental structural differences and there are different basic emotions in various emotion spaces [21]. In view that arousalvalence is the most widely used, we applied this model as the emotion space in this research, see Figure 3.
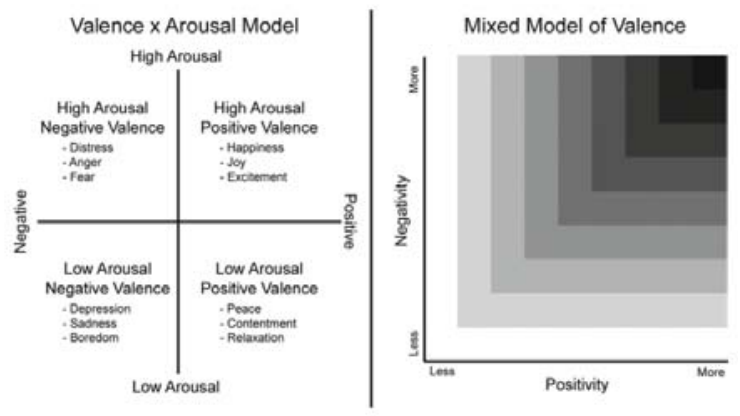

Fig. 3. The Arousal-Valance emotion model

\section{B: Emotion Stimuli Materials}

Music could evoke emotion. Affective music clips were selected as the stimulation materials in this experiment. A collection of 360 instrument music clips were used for music listening session. The music clips was in .wav format with 
sampling rate of $16 \mathrm{KHZ}$, which could reserve most of the music information to insure listening experience. The collection was divided into 6 groups, while each group consisted of 60 music clips (15 happy songs, 15 sad songs, 15 smooth songs and 15 songs in tension emotion. Then a balanced stimuli material set covering all four quadrants of arousal-valence space was insured.

\section{$C$ : Music Listening Experiment}

The experiment was processed as following steps:

- 30 volunteers (aged from 20 to 32) were invited to participate in the experiment. 6 volunteers had music training background, while the other 24 volunteers had no music training background;

- They were arranged in a silent and comfortable room with a constant fluorescent light, facing a 21 inches screen, an ordinary scenery view picture is displayed during the experiment;

- Baseline record session: the volunteer wore the earphone and completed calibration on TOBII X2-30, and their PD signal baseline would be recorded for 2 minutes before the listening session;

- Listening session: each volunteer listened to one group of 60 music clips, with a 5 seconds' break between each clip, the whole listening process lasts for 15 minutes;

- Emotion labeling session: the volunteer was asked to do the emotion labeling work on the clips immediately in 0 to 1 scale for both arousal and valence dimension according to their listening experience after listening session, the labeling questionnaire is shown in Figure 4.
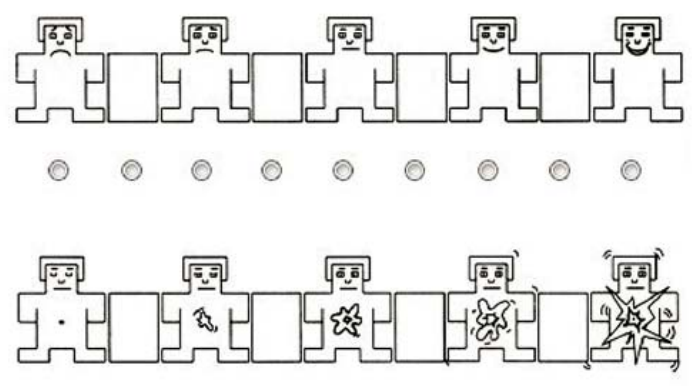

$\circ 00000000$

Fig. 4. Emotion labeling questionnaire

\section{SIGNAL PROCESSING}

Wavelet transform approach was utilized in pupil diameter variation signal processing. Signals were decomposed by 6 levels of Db5 wavelet transform. The decomposed process is presented as Figure 5. Then statistic methods was applied to obtain the the values of Max, Min, Mean, Range, Std, Median, MedAd, MeanAD(Mean Absolute Deviation) of the denoised signals. By means of wavelet transform, a total of 192 wavelet statistic features and 7 wavelet energy features were extracted and formed the pupil emotion feature database.

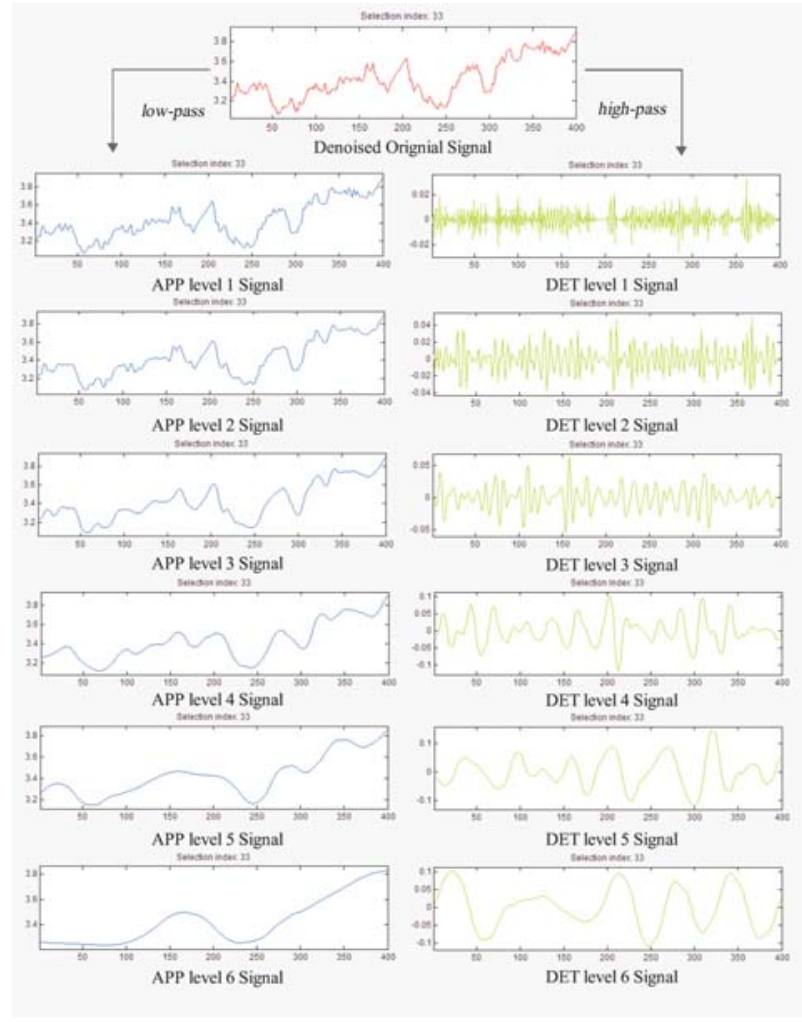

Fig. 5. Signal decompostion by 6 levels of Db5 wavelet transform

\section{EMOTION RECOGNITION MODELING}

We implemented the pupil emotion database with Support Vector Machine method to train the classifier and build the recognition model. In this study, we used LibSVM to build the regression model. In the music listening session, a total of 1751 valid pupil diameter variation signals were obtained. Then we separated the dataset randomly that 1401 instances formed the training set and the other 350 instances formed the testing set. The training and testing results are shown in Table 1.

Table 1: Emotion modeling results based on LibSVM

\begin{tabular}{lcccc}
\hline & \multicolumn{2}{c}{ Training } & \multicolumn{2}{c}{ Testing } \\
\hline & MSE & CC & MSE & CC \\
Arousal & 0.0005 & 0.993 & 0.0072 & 0.614 \\
Valence & 0.0011 & 0.988 & 0.0135 & 0.610 \\
Average & 0.0008 & 0.9905 & 0.010 & 0.612 \\
\hline
\end{tabular}

The best parameters are $\mathrm{c}=2$, and $\mathrm{g}=0.05$, the recognition results shows that the average $\mathrm{CC}$ (Correlation Coefficient) is 0.9905 for training set, a CC value of 0.612 for the testing set, while the average MSE(Mean Squared Error) is 0.0008 for the training dataset and 0.010 for the testing dataset, which is a satisfied result in pupil emotion recognition rate according to the literature studies to our best knowledge. Then the recognition model could be applied in the further application development for MOOCs study communication improvement. 


\section{APPLICATION PROTOTYPE}

At last, we would like to develop an application based on the pupil changes recognition model, then MOOCs students' emotion and status could be recognized and conveyed as a message to teachers, realizing an intelligent communication for both sides. Figure 6 shows the prototype of MOOC Learning Status application interface on cellphone. In the prototype, teacher could adjust teaching method and content according to the response of students' status information visualization, which creates a more indulging and interesting teaching experience.
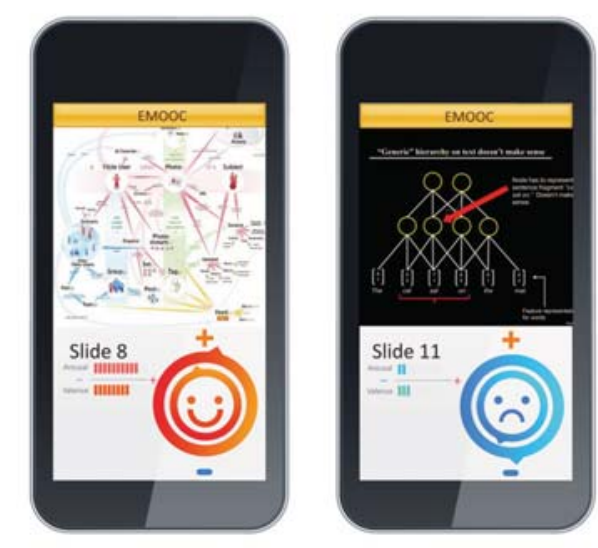

Fig. 6. MOOC Learning Status Application Prototype for Cellphone

\section{CONCLUSION}

In this study, we proposed a communication optimization method targeting to solve the problem of communication barrier of MOOCs between teacher and students. The research needs further in-depth study for system usability and higher recognition accuracy. We have established a model use pupil characteristics to recognize student's studying status. The model's recognition rate for the happy is $64 \%$ and for the sad is $55 \%$. Although the current recognition rate is not very high, but by expanding the data set and optimization algorithm, we can continue to improve our recognition rate. On the existing basis, we can deep learning algorithm to study the larger data sets.

We believed that an intimate MOOCs interactive communication method could be achieved in the future study. Additionally, the application can be implemented to an extended user crowd in MOOCs to achieve barrier free communication, such as the deaf, the elderly, child and people of different first language, since it could help convey status and emotion without words. It is expected that the application could be promoted to a larger range of MOOCs education and improve emotional intelligence and user experience for MOOCs classes.

\section{ACKNOWLEDGMENT}

The author would like to thank Gao Junying and Zhang Lekai for experiment support. This study is partly supported by National Natural Science Foundation of China (61402141), the Fundamental Research Funds for the Central Universities (2014QNA5013), the Foundation of Zhejiang Educational Committee (Y201430757), Zhejiang Province College Students Science and Technology Innovation Activities Plan (2015R407063).

\section{REFERENCES}

[1] Allon, G. Operations management. Chronicle of Higher Education 59, 6 (2012), 10-11.

[2] Belanger, Y., and Thornton, J. Bioelectricity: A quantitative approach. Chronicle of Higher Education (2013).

[3] Breslow, L., Pritchard, D., DeBoer, J., Stump, G., Ho, A., and Seaton, D. Studying learning in the worldwide classroom. research into edxs first mooc. Chronicle of Higher Education 8 (2013), 13-25.

[4] Coursera. Mooc studying, http://www.coursera.com.

[5] Dania, A.-O., Al-Wabil, A., and Hosny, M. Using pupil size variation during visual emotional stimulation in measuring affective states of non communicative individuals. C. Stephanidis and M. Antona (Eds.): UAHCI/HCII 2 (2013), 253-258.

[6] Duneier, M. Teaching to the world from central new jersey. Chronicle of Higher Education 59, 2 (2012), 24-24.

[7] Head, K. Massive open online adventure. Chronicle of Higher Education 59, 34 (2013), 24-25.

[8] Khe, F. H., and Wing, S. C. Students and instructors use of massive open online courses (moocs): Motivations and challenges. Educational Research Review 12 (2014), 45-48.

[9] Kolowich, S. The professors who make the moocs. Chronicle of Higher Education 59, 28 (2013), 20-23.

[10] Koutropoulos, A., Gallagher, M. S., Abajian, S. C., de Waard, I., and Hogue, R. J. Emotive vocabulary in moocs: Context and participant retention. European Journal of Open,Distance and E-Learning 1 (2012).

[11] Matiko, J., Beeby, S., and Tudor, J. Fuzzy logic based emotion classification. In 2014 IEEE International Conference on Acoustics, Speech and Signal Processing (ICASSP), IEEE (2014), 4389-4393.

[12] Onorati, F., Barbieri, R., Mauri, M., Russo, V., and Mainardi, L. Reconstruction and analysis of the pupil dilation signal: Application to a psychophysiological affective protocol. In Engineering in Medicine and Biology Society (EMBC), IEEE (2013).

[13] Partala, T., and Surakka, V. Pupil size variation as an indication of affective processing. International Journal of Human Computer Studies (2014), 4389-4393.

[14] Roth, M. My modern experience teaching a mooc.

[15] Dionisio,D. P, Granholm E, Hillix W. A, Perrine W. F. Differentiation of deception using pupillary responses as an index of cognitive processing. Psychophysiology. 2001,38(2): 205-211.

[16] Avinash R. Vaidya, Chenshuo Jin, Lesley K. Fellows. Eye spy: The predictive value of fixation patterns in detecting subtle and extreme emotions from faces. Cognition 133 (2014) 443-456.

[17] Gross JJ. Emotion and emotion regulation. In L.A.Pervin, O.P.John (Eds.).Handbook of Personality: Theory and Research (2nd.Ed.).New York: Guilford.2000 .

[18] Wei-Chun Chiang, Jeen-Shing Wang, Yu-Liang Hsu. A Music Emotion Recognition Algorithm with Hierarchical SVM Based Classifiers.Computer, Consumer and Control (IS3C), 2014 International Symposium on.2014:10-12.

[19] R. Plutchik, H. Kellerman. Emotion: Theory, research, and experience. New York: Academic. 1980, 3-33

[20] P. Ekman. Emotion in the human face. New York: Cambridge University Press. 1982,39-55

[21] Joseph W. Matiko, Stephen P. Beeby, John Tudor. fuzzy logic based emotion classification. 2014 IEEE International Conference on Acoustic, Speech and Signal Processing (ICASSP) 